\title{
Evaluation of Impinging Stream Vortex Chamber Concepts for Liquid Rocket Engine Applications
}

Huu Trinh, * NASA Marshall Space Flight Center, Huntsville, AL, Charles Kopicz,* ERC, Inc., Huntsville, AL,

Brad Bullard,* NASA Marshall Space Flight Center, Huntsville, AL, Scott Michaels, " U.S. Army Missile Command, Redstone Arsenal, Alabama

\section{ABSTRACT}

NASA Marshall Space Flight Center (MSFC) and the U. S. Army are jointly investigating vortex chamber concepts for cryogenic oxygen/hydrocarbon fuel rocket engine applications. One concept, the Impinging Stream Vortex Chamber Concept (ISVC), has been tested with gel propellants at AMCOM at Redstone Arsenal, Alabama. A version of this concept for the liquid oxygen (LOX)/hydrocarbon fuel (RP-1) propellant system is derived from the one for the gel propellant. An unlike impinging injector is employed to deliver the propellants to the chamber. MSFC has also designed two alternative injection schemes, called the chasing injectors, associated with this vortex chamber concept. In these injection techniques, both propellant jets and their impingement point are in the same chamber cross-sectional plane. One injector has a similar orifice size with the original unlike impinging injector. The second chasing injector has small injection orifices. The team has achieved their objectives of demonstrating the self-cooled chamber wall benefits of ISVC and of providing the test data for validating computational fluids dynamics (CFD) models. These models, in turn, will be used to design the optimum vortex chambers in the future.

Measurements of thrust, pressure, and temperature at several locations on the chamber wall are conducted during hot-fire tests.' For the current chamber configuration, test results show that for the original unlike impinging injector, the chamber wall is relatively cool while the

\footnotetext{
*Aerospace Engineer

Engineer

Copyright 2003 by the American Institute of Aeronautics and Astsronautics, Inc. No copyright is asserted in the United State under Title 17, U. S. Code. The U. S. Government has a royalty-free license to exercise all rights under the copyright claimed herein for Governmental purposes. All other rights are reserved by the copyright owner.
}

thrust efficiency is low. On the other hand, the chasing injectors provide high thrust performance with hot chamber walls.

\section{INTRODUCTION}

Rocket-based combined-cycle engines (RBCC) being considered at NASA for future-generation launch vehicles feature clusters of small rocket thrusters as part of the engine components [1]. Depending on specific RBCC concepts, these thrusters may be operated at various operating conditions including power level and/or propellant mixture ratio variations. To pursue technology developments for future launch vehicle, MSFC is examining vortex chamber concepts for the subject cycle engine as well as small thruster chamber applications. Past studies indicated that the vortex chamber schemes potentially have a number of advantages over conventional chamber methods. Due to the nature of the vortex flow, relatively cooler propellant streams tend to flow along the chamber wall. Hence, the thruster chamber can be operated without the need of any cooling techniques. This vortex flow also creates strong turbulence, which promotes the propellant mixing process. Consequently, the subject chamber concepts not only offer system simplicity, but they also could enhance combustion performance. The test results showed that the chamber performance was markedly high even at a low chamber length-todiameter ratio (L/D) [2]. This incentive can be translated to a convenience in the thrust chamber packaging.

Vortex chamber concepts for bi-liquid propellant systems were first introduced in 1960 [2]. In this early system, one of the propellants is injected tangentially to the inner chamber diameter. The tangential injection develops a rotational flow pattern along the chamber wall. The second propellant is introduced either tangentially at the periphery or radially at the center of the chamber. This injecting scheme creates vortex 
flows, which serve the function of breaking up, vaporizing, and mixing propellants. Hot-fire tests for a small vortex thruster were conducted. The results showed that excellent combustion efficiencies, as high as $98 \%$, were obtained. The wall heat transfer flux with the vortex injectors was significantly lower than with their conventional counterpart impinging injectors. These investigations focused on missile applications, in which hypergolic propellant systems were employed. These efforts were then extended to demonstrate a throttleability feature in conjunction with the utilization of this concept. A 10:1 throttling was accomplished by varying the injection areas of both propellants simultaneously. An average of $94 \%$ in combustion efficiency was recorded over the throttling range [3]. Later, a more rigorous study on the throttleable vortex engine concept was performed with a variety of hypergolic liquid propellant systems [4].

Deep throttling (50 to 1) with a low L/D vortex chamber was demonstrated by Wilson in 1970 [5]. Wilson investigated various injection schemes for hypergolic liquid as well as gel propellant systems. He selected a tangentialtangential injection arrangement for his study since this injector configuration was simple in fabrication and it was able to deliver a specific impulse (Isp), which exceeded $90 \%$ of the theoretical predicted value. In this injection technique, both propellants were injected along the chamber wall and tangential to the inner wall diameter. Both propellant orifices were on the same chamber cross-section plane. Wilson was able to achieve a wide range of throttling using a displacement venturi system. He also observed that the torque force created by the tangential injection was significantly smaller than the axial force.

A recent investigation of the low $\mathrm{L} / \mathrm{D}$ vortex chamber concept for gel propellants was conducted by Michaels [6]. He used both triplet (two oxidizer orifices and one fuel orifice) and unlike impinging schemes to inject propellants tangentially along the chamber wall. The test results showed that excellent performance, with an Isp efficiency of $92 \%$, can be achieved.

Although most of previously discussed studies suggested that the vortex chambers provided relatively cool flows in the near wall region, the hot-fire tests were conducted either with watercooled chambers or at short testing durations.
Ablative materials were sometimes used for critical thruster components, such as the faceplate at the head end, throat, and nozzle sections. To achieve the advantage of the cold wall feature of the vortex chambers, innovative vortex injection schemes were examined at Orbital Technologies Corporation [7]. By injecting the oxidizer tangentially at the chamber convergence and fuel axially at the chamber head end, Knuth et al. were able to keep the wall relatively cold. This concept was employed in tests at which gaseous oxygen and hydrogen at near-stoichiometric mixture ratios were burned in plexiglas chambers. The posttest inspection showed no appearance of overheating on the plexiglas chambers.

This paper reports the results of the efforts at MSFC to examine the ISVC and its alternate configurations with a small LOX/RP-1 thrust chamber. Thrust chamber performance and thermal wall compatibility are evaluated. The chamber pressures, wall temperatures, thrust, and exhaust plume flow fields are measured as appropriate. The test data are being used to validate CFD models, which, in turn, will be used to design the optimum vortex chambers.

\section{MODULAR WORKHORSE VORTEX THRUST CHAMBER}

A vortex test article was designed to be modular so that various injector configurations could be accommodated. When testing different injector configurations the injector module can be replaced. Fig. 1 shows the vortex chamber assembly.

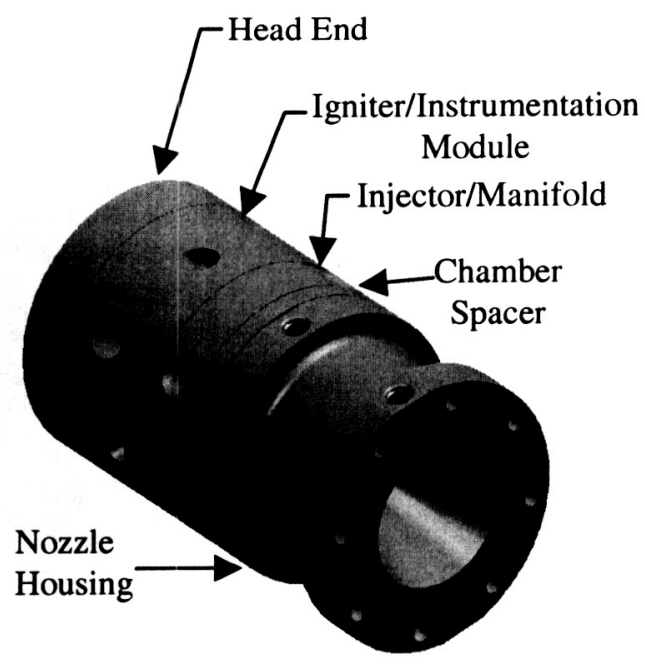

Fig. 1. Vortex Chamber Assembly. 
The vortex chamber assembly consists of (leftto-right) the chamber head housing, igniter /instrumentation module, injector, spacer, and brazed nozzle assembly. All components are constructed of Inconel 718 with the exception of the brazed nozzle assembly, which consists of a 304L stainless steel housing and an OFHC copper nozzle insert. Each component utilizes spring-energized $\mathrm{C}$-seals for fluid isolation. All interfaces contain an outboard seal while the injector and spacer have two additional inboard seals to isolate the manifolds. The entire assembly is clamped together via eight bolts.

The chamber head housing provides instrumentation access for both pressure and temperature measurements. For this study, triethylaluminum and triethylboron (TEA-TEB) liquid mixture is used to ignite the LOX/RP-1 propellant system. The igniter module contains four TEA-TEB ports, angled down toward the injector, which initiate hypergolic ignition upon contact with LOX at startup. Two additional pressure ports are also located in this section. The injector is situated between the igniter housing and the spacer. Fuel and oxidizer manifolds are formed at the interface of the injector with both of the adjoining parts. The primary purpose of the spacer is to thermally isolate the LOX manifold from the nozzle, preventing freezing of the water coolant in the nozzle housing. The brazed nozzle assembly has a 72 degree convergent half-angle and contains 22 coolant channels which feed water from the chamber to the exit nozzle.

It should be noted that the original configuration of the vortex chamber had a slightly different assembly than the present one. The original configuration utilized a gaseous oxygen/hydrogen torch igniter mounted at the chamber head end. A test failure occurred during a first hot-fire test with RP-1. The test article was then redesigned. The cutaway of the final configuration is shown in Fig. 1. The failure investigation [8] was reported and will be discussed in detail in future NASA technical reports.

\section{TANGENTIAL INJECTOR DESIGN}

For this investigation, three injector configurations were designed. The first design was an unlike, impinging-stream injector (designated as injector \#1) where fuel and oxidizer are introduced tangentially at the surface of the chamber wall. This configuration was based on the Army's unlike impinging injector [6] in that the fuel was injected at a downward angle from above (closest to the chamber head end) while the oxidizer angled up from below. The oxidizer was intentionally located in the aft position in order to minimize the potential of accumulating oxygen at the head end of the chamber. The axial momentum of each propellant was equivalent by design, resulting in no net axial component after the streams meet. The injector layout is shown below in Fig. 2.

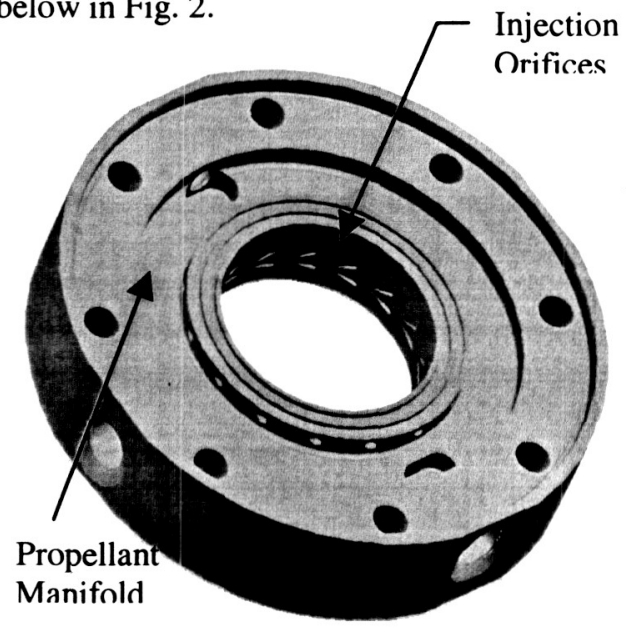

Fig. 2. Unlike impinging injector module.

The remaining two configurations, identified as chasing injectors, were based on the Army's alternating stream concept. In these designs, fuel and oxidizer are injected in the same axial plane, but the propellants alternate around the perimeter. Element orifices are drilled such that the streams impinge approximately 0.2 inches away from the chamber sidewall, in contrast to the design described previously where propellants are tangential to the wall. Although this layout provides less potential for wall cooling, CFD models predicted greatly improved performance. The first of the two chasing injectors (injector \#2) was designed to operate at the same manifold pressure as the unlike impinger, whereas the element orifices in the second chasing design (injector \#3) were reduced, requiring higher manifold pressures and, in turn, producing increased injection velocity and momentum. The chasing injector hardware is shown below in Fig. 3. 


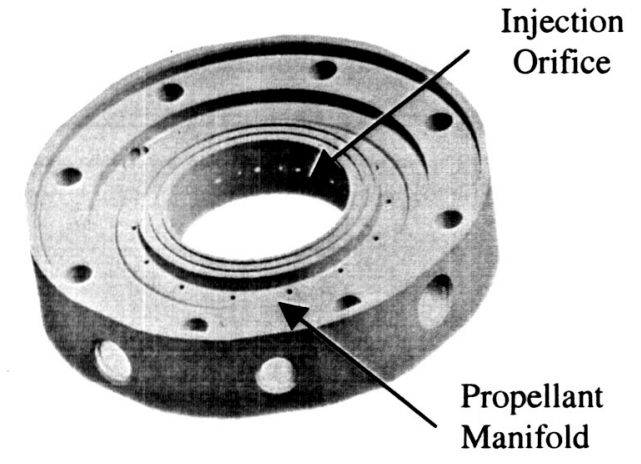

Fig. 3. Chasing injector drawing.

\section{INSTRUMENTATION/MEASUREMENTS}

Chamber pressure was monitored in five locations using static pressure transducers. The chamber was mounted vertically with the nozzle exit facing the ground. One pressure port was 1" above the injector; a second port was 1 " below the head plate. Three pressure measurements were made through the chamber head plate: near the wall, on the chamber axis, and on the mid radius. Each transducer was connected to the chamber via a 7-inch length sense line of 1/4" diameter.

Temperature measurements were made in the same locations as the pressure measurements. The tubing tees allowed $1 / 16^{\prime \prime}$ or $1 / 8$ " sheathed type- $K$ thermocouples to be used to measure temperatures near the chamber wall. Coolant water temperature was measured using type- $K$ thermocouples. One measurement was made prior to water entering the coolant channel manifold, and a second measurement was made just after water exits the nozzle channels. In addition, several accelerometer blocks were mounted on the chamber to monitor the dynamic load.

For the thrust measurement system, three $S$ Beam load cells were used for this setup. The load cells were connected to the head plate of the chamber at 120 degree increments. The load cells were then bolted to a 18 inch wide by 30 inch long by 6 inch deep steel plate welded to the test stand. The plate provides ample mass for the load cells to react against. The design of the load cells is such that only vertical loads are detected.

Measurement of combustion species in the exhaust plume was attempted in this investigation. An emission/absorption measurement system, which is composed of a glow bar, laser spectrometer, and computer for controlling the system and storing the data, was designed and set up. Four attempts at emission/absorption measurements were made throughout this test program, with qualitative carbon-dioxide species measurements collected from two of these attempts.

\section{DESIGNED TEST CONDITIONS}

Although the goal of this project is to obtain data at the main-stage conditions, the facility was designed to include propellant bypass lines for accommodating three levels of propellant mass flow rates, as shown in Table 1. These three levels are used to reduce extreme pressure surge during the combustion startup and shutdown as well as to obtain performance data at various propellant flow rates and mixture ratios. For igniting the chamber, TEA-TEB was injected into the chamber right after LOX injection. The LOX/TEA-TEB mixture ratio of 19.6 is used for this study.

Table 1: Propellant levels for test conditions.

\begin{tabular}{|l|l|l|l|l|}
\hline Level \# & $\begin{array}{l}\text { RP-1 } \\
\text { Mass } \\
\text { Flowrate } \\
\left(\mathrm{lb}_{\mathrm{m}} / \mathrm{sec}\right)\end{array}$ & $\begin{array}{l}\text { LOX } \\
\text { Mass } \\
\text { Flowrate } \\
\left(\mathrm{lb}_{\mathrm{m}} / \mathrm{sec}\right)\end{array}$ & $\begin{array}{l}\text { Mix. } \\
\text { Ratio }\end{array}$ & $\begin{array}{l}\text { Theor. } \\
\text { Pressure } \\
(\mathrm{psia})\end{array}$ \\
\hline 1 & 1.28 & 0.49 & 2.6 & 450 \\
\hline 2 & 1.28 & 1.28 & 1.0 & 570 \\
\hline 3 & 2.8 & 1.07 & 2.6 & 1000 \\
\hline
\end{tabular}

\section{TEST FACILITY}

The test facility provided RP-1, liquid oxygen, TEA-TEB, and water for testing the vortex chamber. The propellant tanks are rated to 3000 $\mathrm{psi}$, although the relief valve settings limited delivery pressure to a 2700 psi maximum. RP-1 and liquid oxygen flows were controlled by a cavitating venturi. The TEA-TEB flow was controlled by an orifice. Deionized water was used for nozzle cooling. Gaseous nitrogen purged all the test systems.

The losses in the facility were characterized by cold flow testing. Loss coefficients were recorded in a spreadsheet for use in $1 D$ pre-test and post-test calculations. Initial testing with LOX and TEA-TEB and tests injecting RP-1 were conducted to confirm facility and ignition characteristics. 


\section{RESULTS}

\section{Difficulty in combustion startup transient:}

Due to the nature of flow vortices developed from the vortex chamber concept as well as a potential of freezing RP-1 when mixing with cryogenic LOX, considerable efforts have been dedicated for fine-tuning the engine startup. A LOX/TEA-TEB mixture ratio range from 35 to 45 is normally used for igniting a conventional LOX/RP-1 combustion chamber. However, through a series of ignition tests, it was determined that a mixture ratio of 20 should be used so that the chamber wall temperature is adequately high enough to reduce the chance of freezing RP-1 droplets when they are injected at the later startup stage.

Through considerable data reviews, the results suggested that the vortices tend to partially fill the pressure and temperature sensor lines on the chamber wall with propellants at the initial startup stage. Ignition of the propellants in the lines momentarily has created random pressure and temperature spike readings from these lines. Thus GN2 purge has been used for some sensor lines to eliminate test cut-off due to exceeding measured pressure limits.

The workhorse chamber was designed in a modular format and C-seals are used at the joint surfaces of these modules. Flow circulations along the chamber wall created by tangential propellant injection could carry combustible fluid into these joint gaps. Consequently, combustion sometimes occurs in these gaps and causes some damages of the seals and the joint surfaces. These issues were observed on several tests. Hence, a borescope was extensively used for visual inspection after each test. If the inspection indicates the need of further inspection and potential hardware repair, the test article would be disassembled for replacing the seals and for resurfacing the joints. Throughout 34 hot-firings in all test series, only one repair of the joint surface was performed. A higher chamber bolt torque value was applied to reduce the gap size and the problem was subsequently eliminated.

In addition, it was also speculated that initial pressure spikes might also be created by having residual RP-1 in the fuel line. The residual RP-1 accumulates from previous tests. Removal of a portion of the RP-1 supply lines to allow inspection for RP-1 residual and cleaning it out as necessary was also implemented in the test procedure. Additional efforts, such as reduction in the propellant-filled volume and fine-tuning of valve open/close timing have also been made to reduce the propellant priming time.

\section{Vortex chamber hot-firings:}

Thirty-four hot-fire tests of the vortex chamber are conducted in this study. Figure 4 shows the last hot-fire test. Considerable ignition tests were carried out with the first injector to resolve issues on the engine startup transient. Once the startup conditions are well understood, only a few tests for each injector are required. Some later tests are run up to 20 seconds in an attempt of collecting the exhaust plume species data.

\section{Theoretical performance calculation:}

Although the primary objective of this effort is to demonstrate the vortex chamber concept and to provide test results for validating the CFD vortex chamber models, performance of each configuration has also been evaluated by comparing the theoretical thrust and characteristic velocity $\left(C^{*}\right)$. A widely-used TwoDimension Kinetic (TDK) computer program has been used to calculate the theoretical thrust and $\mathrm{C}^{*}$ values. A boundary layer module in the code was also activated. Hence, performance losses due the wall viscous effect and the twodimensional divergence theoretically are removed. So the combustion chamber efficiencies can be reasonably predicted. 


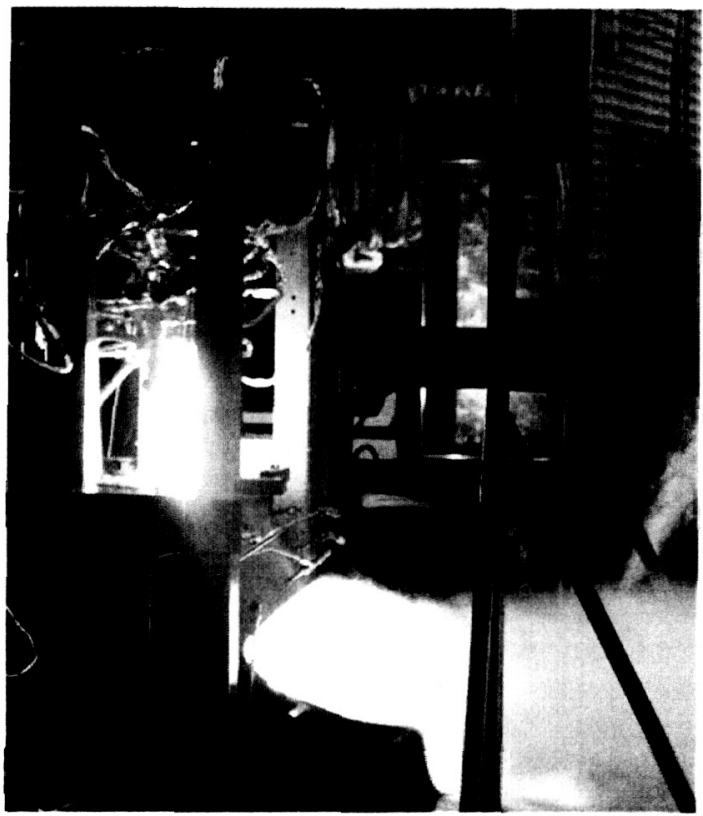

Fig. 4. Hot firing of vortex chamber.

Test Results of the thrust chamber performance:

The test results showed inherent non-uniform pressure and temperature distributions that exists in the chamber. Pressure measurements vary along the chamber wall and across the head end and exhibit a decrease from the injector to the head-end chamber and the outer wall to the centerline. This general trend suggests that the flow field might circulate upward along the wall and direct inward on the chamber head end. Finally, it entrains into the enter core and exits to the nozzle. Preliminary CFD results also show this flow field structure.

For the performance aspect, the $\mathrm{c}^{*}$ efficiency, in general, was observed to be higher than the thrust efficiency for all of the injector configurations. Some propellant swirling along the chamber wall is speculated to have exited through the throat. The presence of the liquid propellant in the throat would reduce the effective throat area. A smaller throat area would require less propellant to be burned to achieve the expected chamber pressures. A portion of the propellant ejected through the nozzle unburned reduced the available energy to generate thrust.

The unlike impinging injector (injector \#1) was tested at several chamber pressures and mixture ratios. Figure 5 shows normalized $C^{*}$ efficiency of injector \#1. as several LOX/RP-1 mixture ratio (MR). For this particular configuration, it appears as the maximum combustion efficiency is at about $M R=1$. The same trend is also observed in the thrust efficiency as shown in Fig.6.

Fig. 7 shows a comparison of the $C^{*}$ efficiency of the three injector configurations at MR 1. The results show an improvement of the $\mathrm{C}^{*}$ efficiency for the chasing injectors, injector \#2 and injector $\$ 3$.

For the wall temperature comparison, the chamber wall is relatively much cooler for the unlike impinging injector as compared to the chasing injectors. It should be noted that injector 2 and injector 3 were tested at reduced propellant mixture ratio. When the chamber was tested using injectors 2 and 3 at the desired mixture ratio of 2.6, the wall temperatures were extremely high, and the material of the test chamber would not tolerate prolonged exposure to that temperature. As a result injectors 2 and 3 were tested near a mixture ratio of 1.0 to keep the wall temperatures within chamber material limits.

\section{INSTABILITY}

The test data of all hot firings are reviewed for examining combustion instability. The results indicate that although some random pressure spikes occur on some tests, only one test for a second injector showed a fluctuation in pressure at a fundamental frequency of $510 \mathrm{~Hz}$ and its harmonics across the entire spectrum. The high amplitude oscillation was present on all the pressure transducers, accelerometers, and load cells. A pressure trace from this test is shown in Fig. 8. At test shutdown, a low peak fluctuating pressure was evident on a static pressure transducer. Its value was approximately $50 \%$ of the mean chamber pressure.

The instability is most likely chug instability because of its low frequency, linear characteristics, and the possibility of gas entrapped in the propellant flow. An analysis of the oxygen condition in the manifold at the time the instability begins to grow showed the oxygen was in transition from a gaseous phase to a liquid phase. 


\section{CONCLUSION}

Due to the nature of flow vortices developed by the ISVC and a potential of freezing RP-1 by mixing with cryogenic LOX, considerable efforts have been focused on the combustion startup. The valve open/close timing, propellant priming times, and propellant mixture during this period are carefully fine-tuned. For all three injectors studied, only the unlike impinging injector provides the relatively cooled flow on the chamber wall. High combustion performance was achieved from the other two chasing injectors; however, this high performance, at least for this chamber configuration, is also associated with high wall temperature. Consequently, these two injectors were only tested at MR 1.

Overall, this effort was not intended to study the optimum vortex chamber concept. The objective was to demonstrate the self-cooled chamber wall feature of the ISVC and to provide data for CFD validation. The CFD analysis and results of comparison between test data and CFD prediction are underway and will be reported in the future. Other future work will be required to modify the injector design and chamber dimensions to obtain an optimum configuration.

\section{ACKNOWLEDGEMENTS}

The author would like to acknowledge the contributions of a number of MSFC personnel and contractors on this project. Cynthia Sprader and her test team have labored many hours to prepare the test facility and to perform the hotfire tests. Neil Myers and George Xenofos have dedicated their efforts to the design and fabrication of the test article hardware. Mike Martin, Matthew Casiano and Tom Nesman have evaluated the test results. Francisco Canabal, Van Luong, and Steve Androlake have conducted CFD prediction, thermal, and stress analyses, respectively. Finally, Robin Osborne of
ERC and Richard Farmer of SECA have spent much time to develop the E/A measurement system and to perform the combustion exhaust plume measurements.

\section{REFERENCES}

[1] Daines, R. and Segal, C., Combined Rocket and Airbreathing Propulsion Systems for Space-Launch Applications, Journal of Propulsion and Power, Vol. 14, No. 5, Sept.-Oct. 1998, pp. 605-612.

[2] Dawson, B., Feasibility Study on Vortex Combustion, Report RMD 1165-Q5, Contract DA-30-069-ORD-2772, Report Period: June 25 - Sept. 24, 1960.

[3] Feigel H. and Fabbro A., Controllable Thrust Chamber Study Program, Task III, Varitex Prototype, Final Report RMD 6020-F, Contract NAS 7-114, Jan. 1963.

[4] Chatfield, D.S., Radially Distributed Annular Combustor For Packaged Liquid Rocket Engines (U), (Radial State of the Art Program), Final Report RMD 6048-F, Contract Now 65-0540-c, April 1967.

[5] Wilson, B., All-Fluid Throttling of The Low L/D Vortex Engine, 12 ${ }^{\text {th }}$ JANNAF Liquid Propulsion Meeting, CPIA Pub. 201, Vol. 1, Oct., 1970, pp. 69-85.

[6] Michaels, R. and Wilson, B., The Low LD Vortex Engine for Gel Propellants, The 1995 JANNAF Gel Propulsion Technology Symposium, Sept. 19-21, 1995.

[7] Knuth W., et al., Vortex Combustion O2/HC Rocket Engine Development, Phase III SBIR Contract with NASA, NAS8-00116.

[8] Dexter, Carol, "NASA/MSFC Army Vortex Incident Review: Final Report," NASA Marshall Flight Center Internal Memo TD61(02-010), May 01, 2002 
$39^{\text {th }}$ AIAA/ASME/SAE/ASEE Joint Propulsion Conference and Exhibit Huntsville, Alabama

July 20-23, 2003

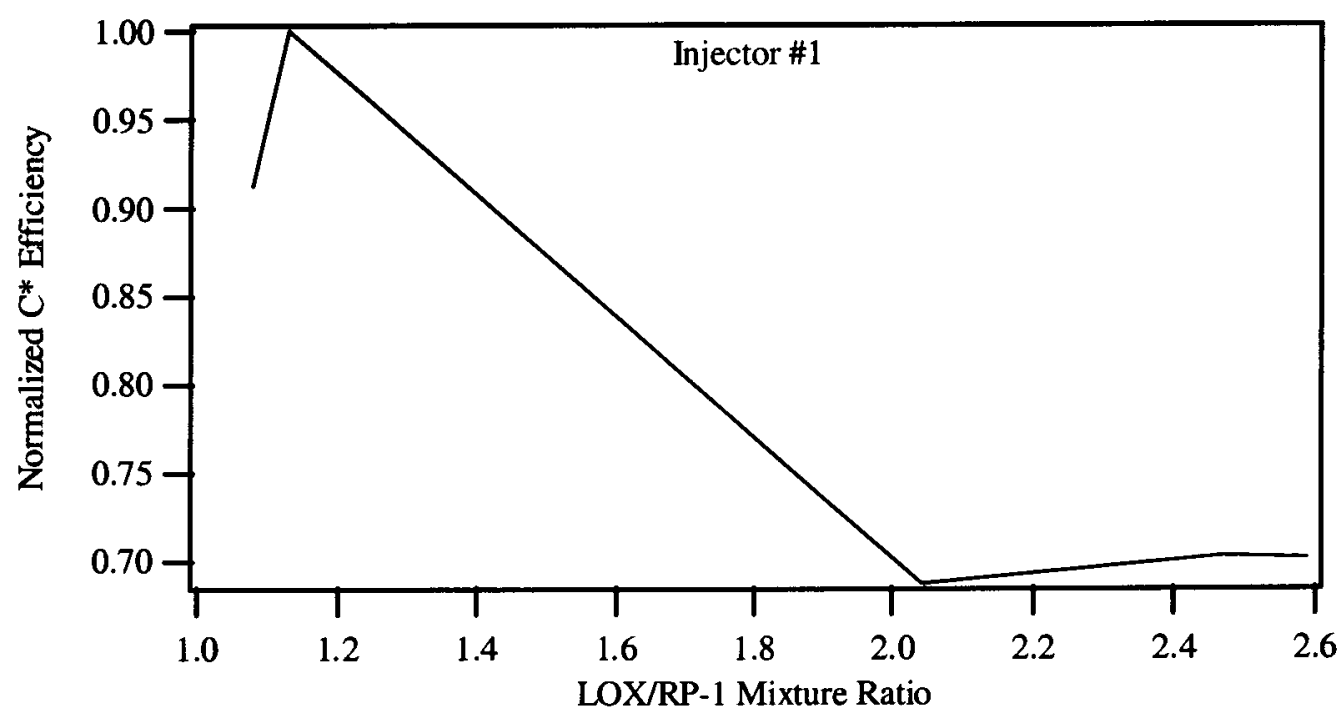

Fig. 5. Change in Normalized C* Efficiency of Injector 1

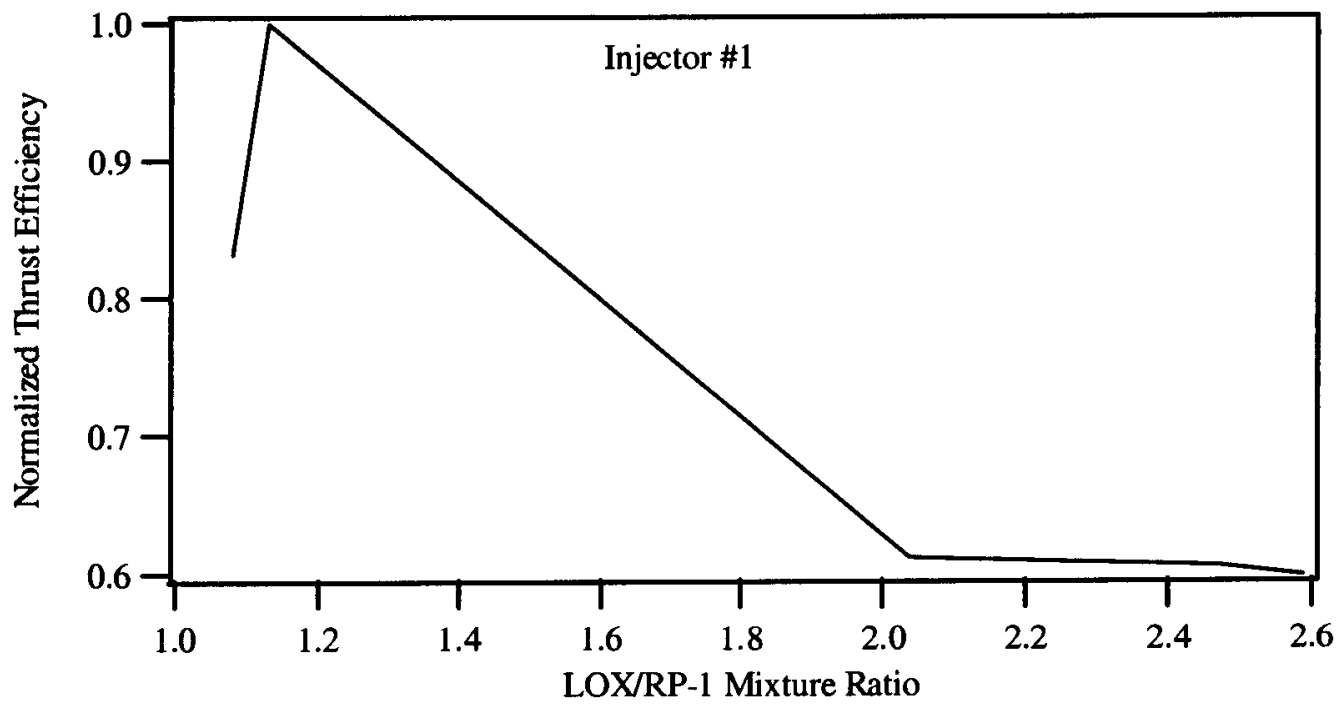

Fig. 6. Change in Normalized Thrust Efficiency of Injector 1 


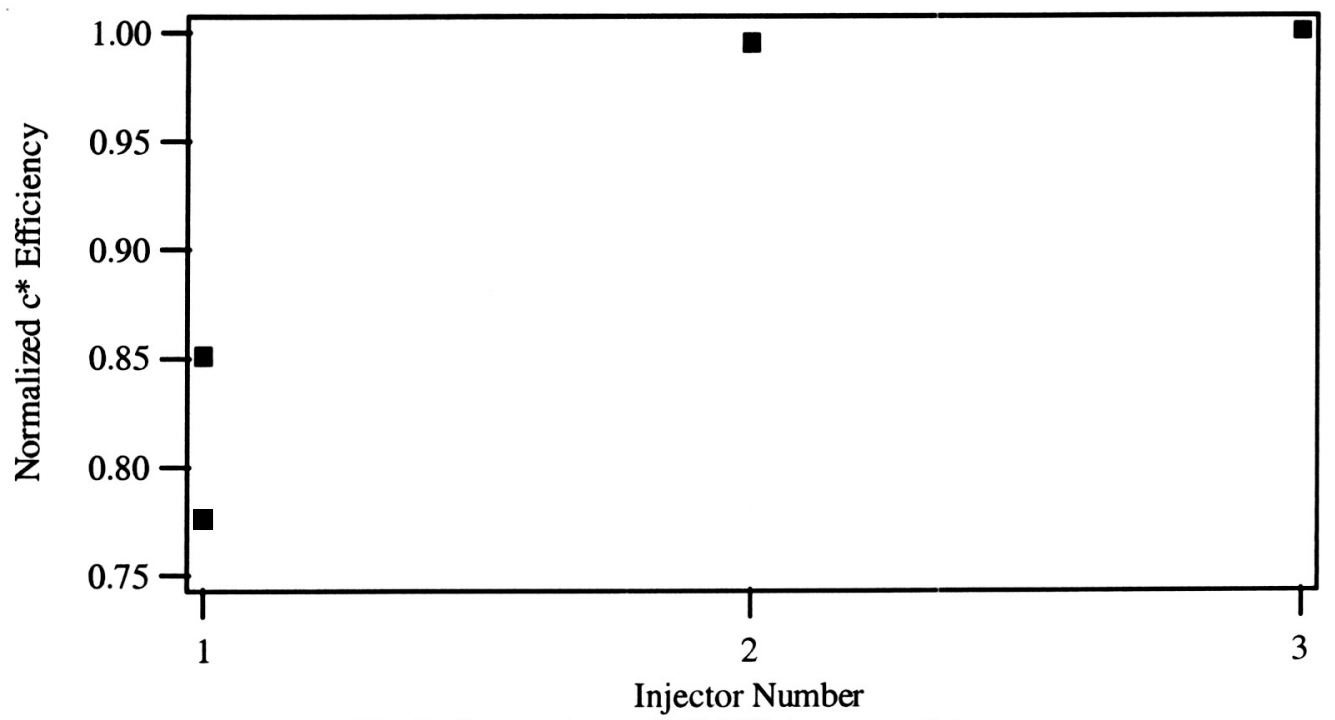

Fig. 7. Comparison of $C^{*}$ Efficiency per Injector

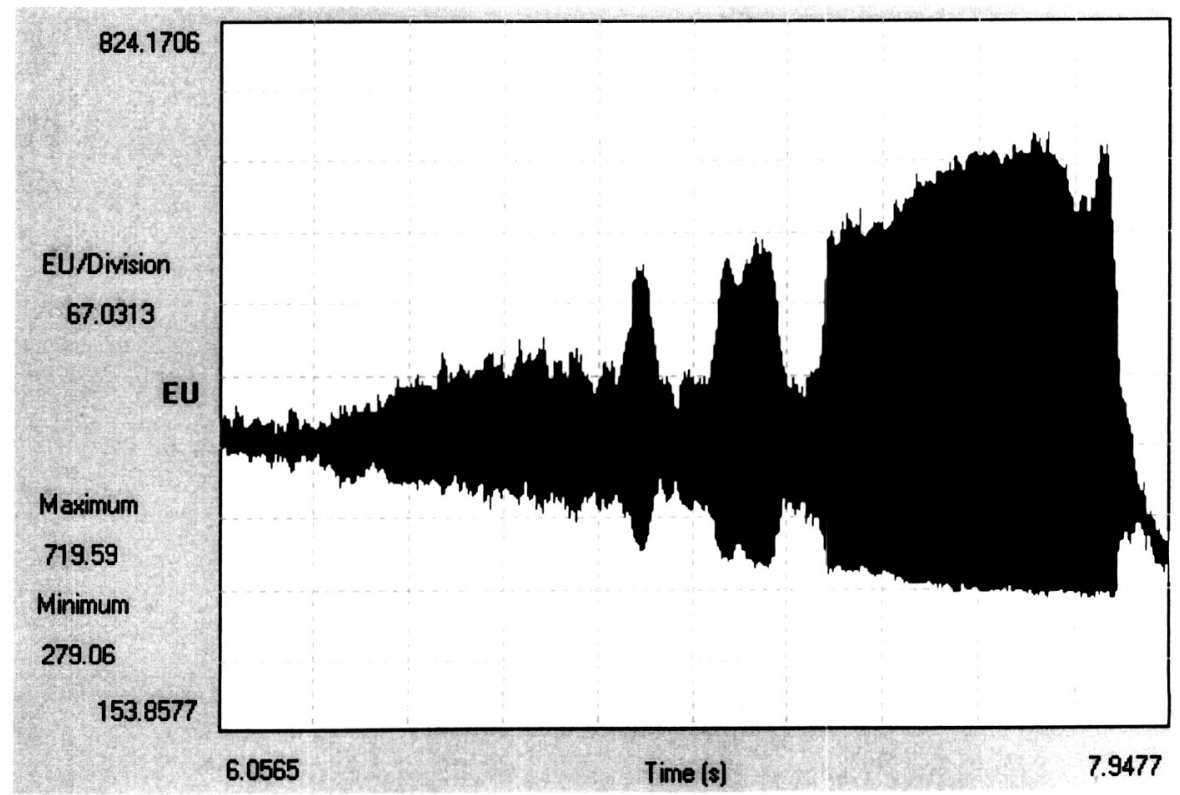

Fig. 8. Unstable Chamber Pressure Response of injector \# 2 in test \#28 